\title{
Spatial Evolutionary Games of Interaction among Generic Cancer Cells
}

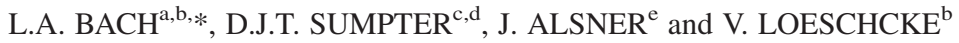 \\ ${ }^{\mathrm{a}}$ EVALife group, Institute of Computer Science, University of Aarhus, Building 540, Ny Munkegade, DK-8000 Aarhus C, Denmark; ${ }^{\mathrm{b}}$ Department of \\ Ecology and Genetics, University of Aarhus, Building 540, Ny Munkegade, DK-8000 Aarhus C, Denmark; ' ${ }^{\mathrm{C}}$ Mathematics Department, Umeå University, \\ SE-901 87 Umeå, Sweden; ' Centre for Mathematical Biology, Mathematical Institute, Oxford University, 24-29 St Giles', Oxford OX1 3LB, UK; \\ ${ }^{\mathrm{e}}$ Department of Experimental Clinical Oncology, Aarhus University Hospital, Aarhus, Denmark
}

(Received 1 November 2002; Revised 31 July 2003; In final form 14 August 2003)

\begin{abstract}
Evolutionary game models of cellular interactions have shown that heterogeneity in the cellular genotypic composition is maintained through evolution to stable coexistence of growth-promoting and non-promoting cell types. We generalise these mean-field models and relax the assumption of perfect mixing of cells by instead implementing an individual-based model that includes the stochastic and spatial effects likely to occur in tumours. The scope for coexistence of genotypic strategies changed with the inclusion of explicit space and stochasticity. The spatial models show some interesting deviations from their mean-field counterparts, for example the possibility of altruistic (paracrine) cell strategies to thrive. Such effects can however, be highly sensitive to model implementation and the more realistic models with semi-synchronous and stochastic updating do not show evolution of altruism. We do find some important and consistent differences between the spatial and mean-field models, in particular that the parameter regime for coexistence of growth-promoting and nonpromoting cell types is narrowed. For certain parameters in the model a selective collapse of a generic growth promoter occurs, hence the evolutionary dynamics mimics observable in vivo tumour phenomena such as (therapy induced) relapse behaviour. Our modelling approach differs from many of those previously applied in understanding growth of cancerous tumours in that it attempts to account for natural selection at a cellular level. This study thus points a new direction towards more plausible spatial tumour modelling and the understanding of cancerous growth.
\end{abstract}

Keywords: Tumour cells; Evolutionary game theory; Individual-based model; Frequency-dependent interaction; Payoff matrices; Cellular interaction

\section{INTRODUCTION}

While there have been many mathematical models of cancerous tumours which attempt to characterise their growth (see for example Qi et al., 1993; Kansal et al., 2000; Sherratt and Chaplain, 2002) few, if any, of these models account for the frequency-dependent selection which acts on the cells inside a cancerous tumour. This situation is in sharp contrast with that in ecology where it is widely acknowledged that the growth of, for example, animal populations are determined both by the processes of diffusion, aggregation, overcrowding, etc. and by the action of natural selection as phenotypes competes. Evolutionary models have previously shown that natural selection may lead to self-limitation or extinction of groups of aggressive phenotypic strategies
(Riechert and Hammerstein, 1983). Such phenomena may also be observable in tumours since the selective process on the cellular level is similar to selection among genotypes in a population of animals or other organisms (Nowell, 1976; Tomlinson, 1993; Mora et al., 2001). Furthermore, Tomlinson et al. (1996) showed that selection can be more important than the increased mutation rate often observed in connection with cancerous growth. Clinical observations such as spontaneous regression of tumour growth (Challis and Stam, 1990; Papac, 1996) give the intriguing idea that models accounting for the frequency-dependent selection could demonstrate that phenomena such as tumour regression can arise through internal selective forces on the tumour itself. Indeed, it is highly plausible that internal evolutionary dynamics could, as is the case in

*Corresponding author. E-mail: lbach@daimi.au.dk 
theoretical ecology, lead to an improved understanding of cancerous growth as a complex adaptive system (Dieckmann, 1997).

Cancer cells in tumours typically show a variety of biochemically mediated mutual influences on cell proliferation. Therefore, local selection regimes, and hence the evolutionary dynamics can strongly depend on the nature of such cellular interactions (Tomlinson, 1997; Tomlinson and Bodmer, 1997). Given the high genotypic diversity found in tumours it may be crucial, in relation to therapy, to understand the evolutionary dynamics within tumours, especially if frequency-dependent cell interactions profoundly affect the dynamics (Bach et al., 2001). Traditional chemotherapy, and to some extent ionising radiotherapy, often operate by inhibiting the nucleic acid synthesis of the cells, which in turn mainly affect the exceedingly proliferating cells (Boyer and Tannock, 1998). Unfortunately, a well-known disadvantage of such an approach is the detrimental effect on those healthy cell types that naturally divide at a high rate. During the last decade, however, our understanding of the genetic abnormalities underlying the development of cancer has improved dramatically (Hanahan, 2000). Together with the recent reports of the first draft of the human genome sequence and the development of a large number of novel technologies to analyse it, a new field - pharmacogenomics - has emerged (Onyango, 2002). One of the aims of pharmacogenomics is to design drugs for molecular specificity providing a high degree of genotype specific selection pressure.

Interaction metaphors such as the Dilemma games, the Hawk-Dove game, and the Ultimatum game have been widely used to model evolution of co-operation and frequency-dependent selection in evolutionary ecology (Maynard-Smith and Price, 1973; Axelrod and Hamilton, 1981; Hofbauer and Sigmund, 1988). The same way of accounting for interactions may be exploited when describing the behaviour of interacting units on lower hierarchical levels. Applying the same basic game theoretic ideas to the growth of cancerous cells, Tomlinson and Bodmer (1997) showed that stable polymorphisms of different cellular strategies can persist, not merely as transient phenomena, but as stable states maintained without requiring external causal explanations (Tomlinson, 1997; Tomlinson and Bodmer, 1997). However, as pointed out by the authors, resting on assumptions of random encounters and perfect mixing the mean-field approximation neglects any local effects and spatial correlations and the cells interact exclusively in terms of global frequencies (Tomlinson and Bodmer, 1997). (For a more general discussion of local effects and discreteness see, e.g. Durrett and Levin, 1994; McGlade, 1999, and references therein.) Allowing for spatial explicitness in, for example, the Prisoner's Dilemma game, the HawkDove game, and more recently the Ultimatum game and the Public Goods game gave highly different results compared to corresponding non-spatial mean-field representations (Nowak and May, 1992; Herz, 1994;
Lindgren and Nordahl, 1994; Killingback and Doebeli, 1996; Page et al., 2000; Hauert, 2002; Szabo and Hauert, 2002). These models lead to the conclusion that space generally facilitates the invasion and persistence of cooperative strategies because spatial correlations inhibit the possibility for non-co-operators to exploit and outcompete co-operators (see also, Koella, 2000). Moreover, spatial frequency-dependent interactions also seem to favour coexistence when mean-field models tend to predict competitive exclusion (Molofsky et al., 1999).

In this paper, we make a step towards spatial models of cancerous growth that include selective forces. We extend Tomlinson and Bodmer's (TB) model to allow for a greater range of cell-cell interactions and thus encompass many of the types of interactions seen in real tumours. We discuss how these interactions affect cell proliferation in the general context of two-player games. We then employ a range of spatial individual-based, or cellular automata type, models to investigate the effect of spatially local interactions between cells. The effect of stochasticity and the importance of local random drift of the cell genotype frequencies are also explored. Bearing in mind the fixed positions of cells in solid tissue tumours, we believe that the significance of spatial effects in tumour cell interactions is importunately relevant for further progress in tumour modelling. The models, we present, are at this stage far from being predictive medical tools but we see our contribution as a "growth promoting factor" in the further development of mathematical models to understand tumour growth.

\section{MEAN-FIELD EVOLUTIONARY GAME MODELS}

In order to determine the evolutionary dynamics of tumour cells, the different genotypic strategies adopted by the cells can be expressed as a two-player game theory model. One such scenario, presented by Tomlinson and Bodmer (1997), defines a cellular genotypic strategy, hereafter called a "growth promoter" or "co-operator", where a costly growth factor is produced. This factor conveys a benefit to the cell itself as well as to any neighbouring or interacting cells. The alternative strategy is the "nonpromoter" or "defector" strategy. Such cells produce no growth factor, and hence incur no cost, but do benefit when interacting with a growth promoter cell. This model corresponds, for instance, to processes such as neoangiogenesis, which is the aberrant reformation of blood vessels in tumours. Here, the reformation relies on a joint effort in the biochemical stimulation of existing blood vessels to form new vascularisation (Boehm et al., 1997; Carmeliet and Jain, 2000). In such cases cells that do not produce stimulating chemicals may be considered as "defectors", while those producing the chemicals may be thought of as "co-operators".

The payoff matrix for TB model is given in Table Ia. This table defines how the fitness of each of the genotypic strategies depends on its interaction with other strategies, 
TABLE I Payoff matrix modified from Tomlinson and Bodmer (1997)

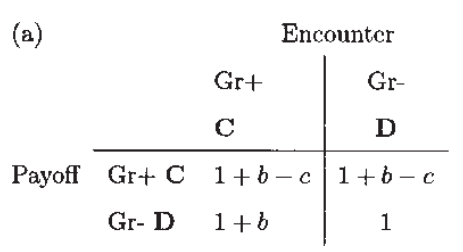
(b) Encounter

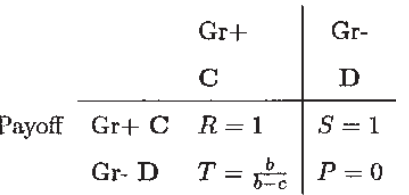

$\mathrm{Gr}+$ and $\mathrm{Gr}-$ are the growth promoting and non-promoting strategies, respectively. In a game theoretical context Gr + corresponds to co-operation (C) and Gr - to defection (D). The values in the matrix describe for a given cell type the payoff obtained depending on the strategy of itself as well as that of the interaction partner it encounters. Note that in the mean-field, such matrix serve as the basis for calculating the mean fitness of a given strategy depending on the composition of the global population described by a single state variable in form of the real value fraction of one of the strategies. (a) Interactions defined in terms of the mutual effects of cancerous proliferation strategies. The parameters describe the benefits $b$ and costs $c$ of a generic growth promoter. A passive non-promoter can also obtain $b$ when it encounters a growth promoter, otherwise it is left with base line proliferation, i.e. a payoff of unity. (b) Identical to (a) but normalised following Hauert (2002) such that $R=1$ and $P=0$.

in terms of the benefit $b>0$ and cost $0<c<b$ of producing the growth promoting factor. Note that the autocrine effect implies that the benefit is always obtained by a growth promoter (even when interacting with nonpromoters, i.e. top right entry of Table I). Such payoff tables can be used to define a replicator equation for the different genotypic strategies (Hofbauer and Sigmund, 1988). The replicator equation determines — in the case of a well-mixed population of cells (i.e. when encounters between cells occur entirely at random) - the evolutionarily stable population of strategies. In the case of the TB model the replicator equation for the number of growth promoting cells, $x_{t}$, is

$$
x_{t+1}=\frac{(1-c+b) x_{t}}{(1-c+b) x_{t}+\left(1+b x_{t}\right)\left(1-x_{t}\right)}
$$

and can be shown to have a single stable equilibrium at

$$
x_{*}=1-c / b
$$

growth promoting cells. The TB model thus predicts, in the well mixed case at least, that growth promoter and non-promoter cells will coexist (Tomlinson and Bodmer, 1997).

The TB model is in fact a specific instance of a general set of two-player games which can be expressed in terms of reward $R$ (C-C interaction), suckers payoff $S(\mathbf{C}-\mathbf{D}$ interaction), temptation $T$ (D-C interaction) and punishment $P$ (D-D interaction). The payoffs for such games can be normalised so that $R=1$ and $P=0$. As a result, each of these two player games may be categorised in terms of the values of $S$ and $T$ (Hauert, 2001, and references therein). The replicator equation for these games is then

$$
x_{t+1}=\frac{x_{t}+S\left(1-x_{t}\right)}{\left(x_{t}+S\left(1-x_{t}\right)\right) x_{t}+\left(T x_{t}+1-x_{t}\right)\left(1-x_{t}\right)} x_{t} .
$$

Table Ib gives $S$ and $T$ for the TB model. In terms of the $S-T$ classification of two-player games, the TB game lies on the boundary between the Leader game (where $S>1$ and $T>1$, see Rapoport et al., 1976; Binmore, 1996) and the Hawk-Dove game (also known as the Chicken game or Biker's dilemma, where $S<1$ and $T>$ 1, (see Binmore, 1996; Svenstrup and Christiansen, 2000 for details).

Since $S=1$ in the TB game, it cannot be considered a particularly general model of growth promoting interactions. Indeed, if we again return to the biological basis for the model and consider how different cell genotypes may promote and inhibit growth we can produce a more general description of the interaction process. Specifically, we can introduce the additional biological observation that growth promoting cells are likely to obtain an additional benefit from interacting with another growth promoting cells, over and above the autocrine self-benefit, $b$. We will denote this extra benefit as $e \geq 0$ (note that $e=0$ gives the original TB model). In defining a more general model, it is also useful to think of the benefit to non-promoting cells as

\begin{tabular}{|c|c|c|c|c|c|c|c|}
\hline \multirow[t]{2}{*}{ (a) } & & \multicolumn{2}{|c|}{ Encounter } & \multirow[t]{2}{*}{ (b) } & & \multicolumn{2}{|c|}{ Encounter } \\
\hline & & $\mathrm{Gr}+$ & GI- & & & $\mathrm{Gr}+$ & Gr- \\
\hline \multirow{3}{*}{ Payoff } & & C & D & & & $\mathrm{C}$ & $\mathrm{D}$ \\
\hline & $\mathrm{Gr}+\mathrm{C}$ & $1+b+e-c$ & $1+b-c$ & Payoff & $\mathrm{Gr}+\mathrm{C}$ & $R=1$ & $S=\frac{b-\mathfrak{c}}{b+\mathfrak{e}-\mathfrak{c}}$ \\
\hline & Gr- D & $1+d$ & 1 & & $\mathrm{Gr}-\mathrm{D}$ & $T=\frac{d}{b+e-c}$ & $\boldsymbol{P}=0$ \\
\hline
\end{tabular}
being a parameter $d>0$ which may be changed

TABLE II Payoff matrix for the general model Gr $+/ \mathbf{C}$ and $\mathrm{Gr}-/ \mathbf{D}$ are growth promoter/cooporator and non-promoter/defector, respectively

(a) Biologically defined interactions in a population consisting of a generic growth promoter, which can obtain the benefits $b$, and $e$ and experience the cost $c$. The nonpromoter will obtain the benefit $d$ from interacting with a growth promoter, whereas interaction with another non-promoter only yields a payoff of unity, i.e. base line proliferation. (b) Identical to (a) but rearranged following Hauert (2002) such that $R=1$ and $P=0$. 
independently of the costs and benefits to the growth promoting cells. This gives payoff Table IIa.

We can now use the payoff Table II, that relates parameters $b, c, d$ and $e$ to $S$ (the $\mathbf{C}-\mathbf{D}$ interaction) and $T$ (the D-C interaction), to determine for the wellmixed case the resultant proportions of growth promoters and non-promoters. We see that when the benefit to a nonpromoter from the chemical compound is greater than the total benefit minus the cost to the growth promoter, i.e. $d>b+e-c$, and the benefit to the growth promoter outweighs the cost it pays, i.e. $b>c$ we have a HawkDove game (i.e. $T>1$ and $0<S<1$ ). The replicator equation for proportion of growth promoting cells is then

$$
x_{t+1}=\frac{\left(1-c+b+e x_{t}\right) x_{t}}{\left(1-c+b+e x_{t}\right) x_{t}+\left(1+d x_{t}\right)\left(1-x_{t}\right)} .
$$

The equilibrium proportion of growth promoters in the case where $b>c$ and $d>b+e-c$ is

$$
x_{*}=(b-c) /(d-e) .
$$

It appears, therefore, that the additional benefit, $e$ does not change the general conclusion that the production of costly angiogenic factors is selected for amongst the cells. However, the equilibrium number of growth promoting cells is increased as the extra benefit increases (MaynardSmith, 1982).

Other models of cell interactions are encompassed by the model in Table II. For example, Tomlinson and Bodmer (1997) also consider competition between cells which produce a factor to prevent programmed cell death in a purely paracrine fashion (i.e. no effect on producer) against cells which produce growth factor in a purely autocrine fashion (i.e. benefit to self but not to neighbours). By defining $b-c<0$ the autocrine strategy now represents a purely "altruistic" act on the part of the growth promoter, as $d$ and $e$ remain constant. We see that $T>1$ but $S<0$, giving the well-known Prisoner's Dilemma game. Here, the interesting result is that although these "altruistic" cells would thrive in a pure population of growth promoters, in the well-mixed case, an invading non-promoting cell would reproduce and take over the entire population. This is seen by finding an equilibrium for the replicator equation (3) when $c>b$. In this case, the proportion of growth promoting cells is $x_{*}=0$ corresponding to the defecting or non-promoting strategy being an evolutionarily stable strategy (Hofbauer and Sigmund, 1988).

\section{SPATIAL EVOLUTIONARY GAME MODELS}

Spatial patterns undoubtedly arise in the growth of cancerous tumours, for the simple reason that offspring cells grow adjacent to the parent cell. It is important, therefore, that spatial effects are accounted for when attempting to determine the growth and changing genotypic composition of tumours. Indeed, the fact that the Prisoner's Dilemma naturally arises out of cell interactions points to the intriguing possibility that spatial arrangements of cells may allow for truly altruistic effects on proliferation. That is, we may see the evolution of purely paracrine cells. The results of Nowak and May (1992) and others show that true altruism can arise in the Prisoner's Dilemma when players are arranged in a two dimensional array (Nowak and May, 1992; Nakamuru et al., 1997). Hauert (2001; 2002) has recently presented an extensive study of general two-player $S-T$ spatial games with nearest neighbour interactions. He has shown that for many games, space has an important role in determining the equilibrium proportion of defectors and co-operators. In general, discrepancies are almost always found between results from the mean-field replicator equation and nearest neighbour interaction models.

We now propose a variety of simple two dimensional lattice simulation models to determine the behaviour of such cell interaction dynamics (Wolfram, 1984; Nowak and May, 1992). Each simulation model begins with a $100 \times 100$ array of cells, a proportion of which are growth promoting (C strategy) and some of which are nonpromoting (D strategy). The basic protocol for updating the cells on each generation is as follows: First, a certain number of cells will be removed from the lattice (the number depends on whether updating is synchronous, semi-synchronous or asynchronous, see below for details). The neighbours of the removed cells will then compete to occupy the empty cells. Each non-empty neighbour to the empty cell will perform a two-player interaction with each of its neighbours and hence receive a payoff. The payoff to reach interaction is determined according to the specified payoff matrix (in our case we use payoff Tables I and II). The obtained payoffs from each interaction are summed to yield the neighbour cell's total payoff or fitness, which in turn is used in order to determine which of the candidate neighbour cells will be selected to reproduce (the reproduction may be deterministic where the cell with the highest fitness reproduces or probabilistic where reproduction is proportional to fitness, again see below for details). The "winning" neighbour cell is subsequently copied into the empty site. Hence, an empty site always becomes re-occupied by the proliferation of the cell which wins a local competition. Figure 1 gives a diagrammatic representation of the update scheme for a single removed cell.

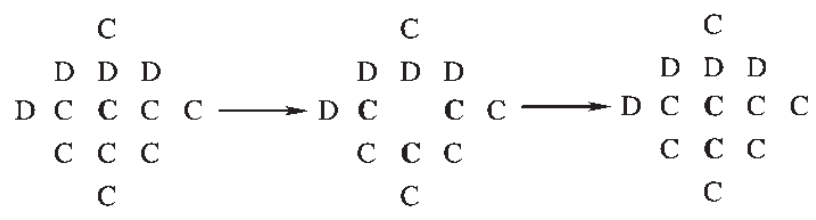

FIGURE 1 A diagrammatic representation of the cell update process. First a cell is selected to be removed (in this case the $\mathbf{C}$ marked in bold in the configuration on the left). Then the four neighbouring cells all assess their fitness (middle configuration). The cell with the highest fitness then reproduces into the empty space (configuration on the right). 
The model description above remains ambiguous. There are now a number of design decisions that must be made in the implementation of lattice simulation models. Results from simulations can be highly dependent on whether updates are synchronous or asynchronous, probabilistic or deterministic, as well as depending upon the size of neighbourhood, or spatial scale, on which cells interact. We thus choose to implement a variety of models and compare the results. These considerations, which may appear at first to be purely technical, will prove to have profound implications for the biological interpretation of our model. We now outline each of the design decisions in detail. Appendix A provides a complete algorithmic description in the form of pseudo code, for each of the simulation models.

\section{Synchronous, Asynchronous or Semi-synchronous Updating}

Synchronous updating means that all the cells die simultaneously, and they are replaced dependent on the strategy of their neighbours before dying. Although synchronous updating is often the choice of implementation in spatial evolutionary game models it presents some problems. Synchronous updating assumes a global controller of the system, which ensures that all sites are updated exactly once in each iteration. This assumption, which gives a very coarse temporal granularity may be violated in a range of natural situations, particularly in the case of cell populations of a considerable size (Huberman and Glance, 1993). Since mortality and the subsequent reproduction of cancerous cells occur sporadically, it is biologically most plausible to have at least some degree of asynchronous updating of cells.

Asynchronous updating of cells means that on each generation a single cell, chosen at random, dies and is replaced. Asynchronous updating presents its own problems, however. The fact that at most one cellular site is updated in any given iteration means that any two adjacent sites can never be updated simultaneously since updating is strictly sequential. This in turn makes the disappearance of small clusters of cells impossible. Phenomena such as local episodes of low oxygen tension giving rise to apoptotic or necrotic areas with several adjacent cells dying necessitates the consideration of how a relaxation of the assumption of strict sequentiality will affect the system.

We therefore, adopt an additional Semi-synchronous updating rule, which does not assume strict sequentiality in the turnover of cells and thus does not suffer the problems of such assumptions. Semi-synchronous updating represents an intermediate and more realistic temporal granularity between the extreme synchronous and extreme asynchronous updating. In the semi-synchronous scenarios presented here individual cellular mortality is 0.1 , i.e. one tenth of the cells die and are replaced on each generation. Thus, with a probability of 0.01 any two adjacent cells will be updated concurrently. This method of updating allows for the biologically realistic situation that occasionally more than one local site is available for exploitation by proliferating neighbour cells.

\section{Neighbourhood Size}

A strong criticism of spatial game theory models is that results can be strongly dependent on the type of neighbourhood rules adopted. Indeed, the thorough investigation of Hauert (2002) showed that very different results are obtained for the von Neumann neighbourhood (where the four adjacent neighbours on a lattice are considered) than for the Moore neighbourhood (where eight adjacent neighbours are considered). We thus, provide simulation results for both of these neighbourhoods, as well as an extended Moore neighbourhood with 24 neighbours (i.e. a Moore neighbourhood with a radius of two cells).

\section{Deterministic or Probabilistic Updating}

Two different schemes of competition for local reproduction are used in order to disclose possible effects of determinism vs stochasticity in the local competition for reproduction. The deterministic updating corresponds to the competitive situation where the "winner takes it all". The score of each individual is compared to all of its neighbours and only the cells with the highest local maximum score are allowed to reproduce. In case of a tie between two competitors a random cell is chosen. This means that whether or not a local cluster configuration of growth promoters will expand or diminish is governed by deterministic rules.

In order to avoid the determinism and discreteness of the "winner takes it all" updating method an alternative implementation was designed. The individual's probability of reproduction is in the probabilistic updating defined in terms of its relative local payoff score. The probability of reproduction is given by the scaled value of own score divided by total score in the neighbourhood. Such local competition allows cellular strategies with lower fitness to have a chance of reproducing, especially when they are locally superior in numbers. In contrast to the deterministic reproduction this implies an effect of local density as to which strategy succeeds in proliferating. A strategy, which in numbers dominates the local neighbourhood can, albeit being inferior in terms of individual payoff, have a higher collective probability of occupying an empty site than, e.g. a single representative of the superior strategy. Inferentially, this effect becomes more pronounced the closer the payoff values of the competing strategies are.

\section{RESULTS}

Semi-synchronous simulations were run for 20,000 generation whereas asynchronous realisations were run 

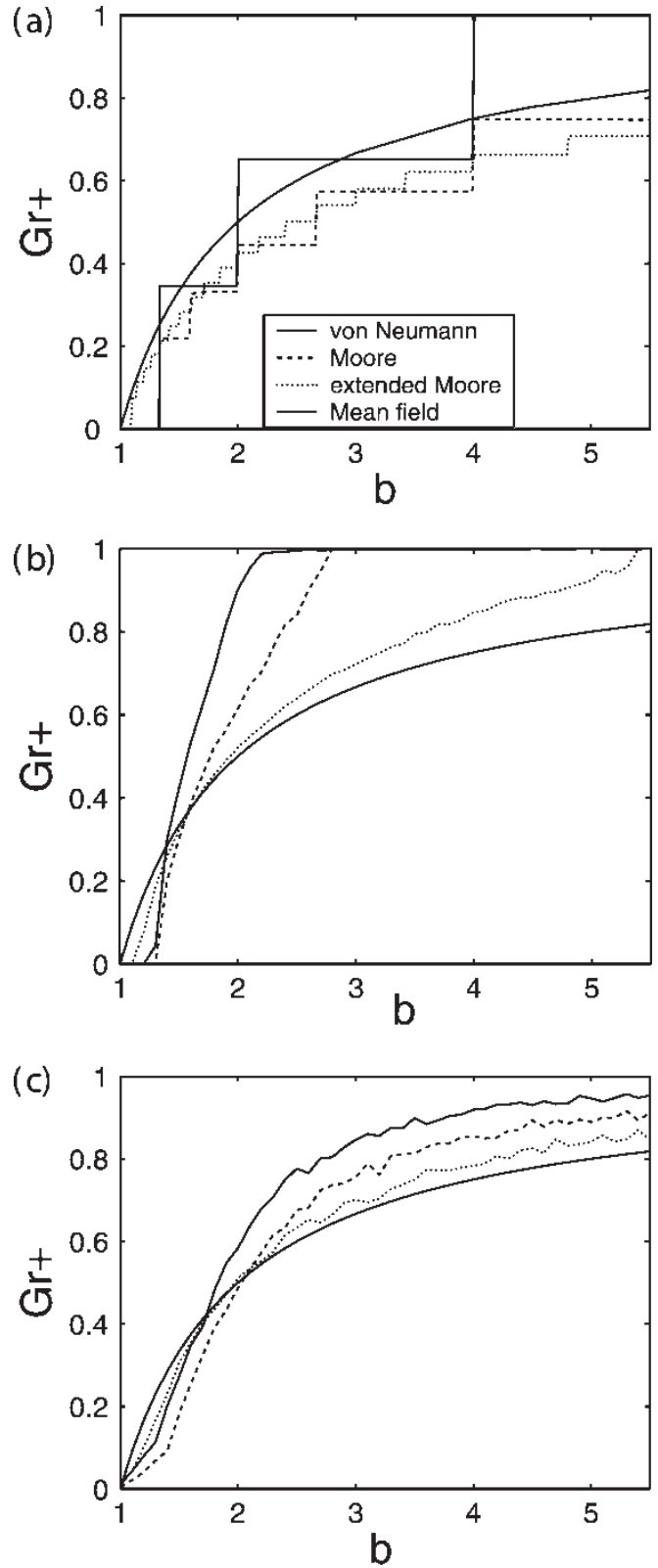

FIGURE 2 (a) Spatial simulation with payoff conditions corresponding to the scenario of Tomlinson and Bodmer (1997) see Table I. The equilibrium proportion of growth promoters $(\mathrm{Gr}+)$ is depicted as a function of the benefit $b$, while $c$ is fixed at unity. Competition for reproduction is fully deterministic and updating is semi-synchronous with individual mortality 0.1 . Thin solid line shows the mean field result according to Eq. (2). Thick solid, dashed, and dotted lines represent simulation results from the von Neuman neighbourhood, the Moore neighbourhood, the extended Moore neighbourhood, respectively. (b) As in Fig. 2a but with probabilistic reproduction. (c) As in Fig. 2a but with probabilistic reproduction and asynchronous updating.

for 200,000 generations in order to ensure that a stable genotype distribution had been reached. The results shown are averaged over 2,000 and 20,000 generations for the semi-synchronous and the asynchronous scenarios, respectively. Figures 2-4 show the effect of parameters $b$ or $d$ on the equilibrium proportion of growth promoters and replicated simulations showed highly consistent and practically identical results (not shown). In the TB and Hawk-Dove scenarios the initial proportion of growth
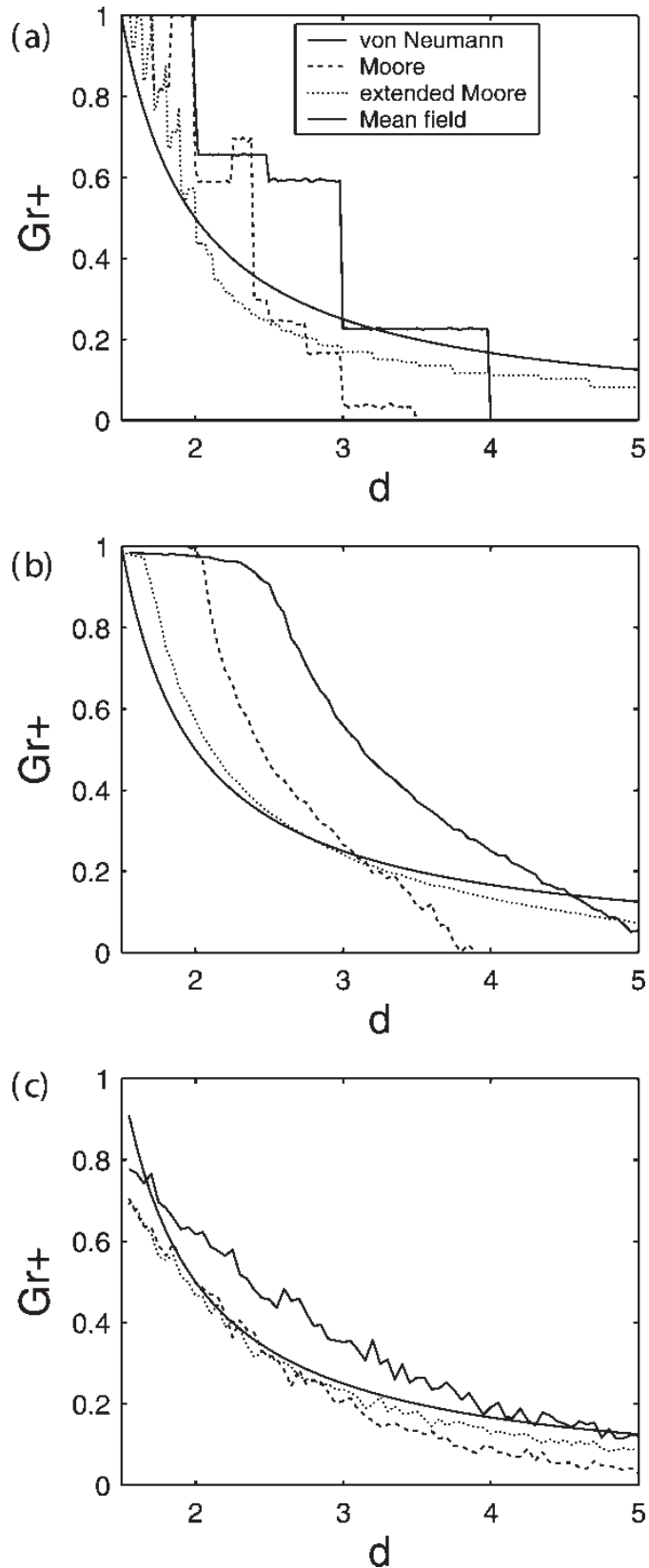

FIGURE 3 (a) Hawk-Dove interaction scenario obtained according to Table II with parameters $b=1, c=0.5$, and $e=1$. The equilibrium proportion of growth promoters $(\mathrm{Gr}+)$ in the population is depicted as a function of increasing values of $d$. Competition for reproduction is fully deterministic and updating is semi-synchronous with individual mortality 0.1 . The population was initiated with a proportion of growth promoters of 0.1. Thin solid line shows the mean field result according to Eq. (4). Thick solid, dashed, and dotted lines represent the von Neuman neighbourhood, the Moore neighbourhood, and the extended Moore neighbourhood, respectively. (b) As in Fig. 3a but probabilistic reproduction. (c) As in Fig. 3a but probabilistic reproduction and asynchronous updating.

promoters was 0.1 . However, other initial conditions were explored (not shown) indicating that the equilibrium proportion of growth promoters was robust to initial conditions, as was the case in the analytical results of the non spatial models. (Coexistence obviously requires the initial presence of both strategies in the population.) Following Hauert (2002), the Prisoner's Dilemma 

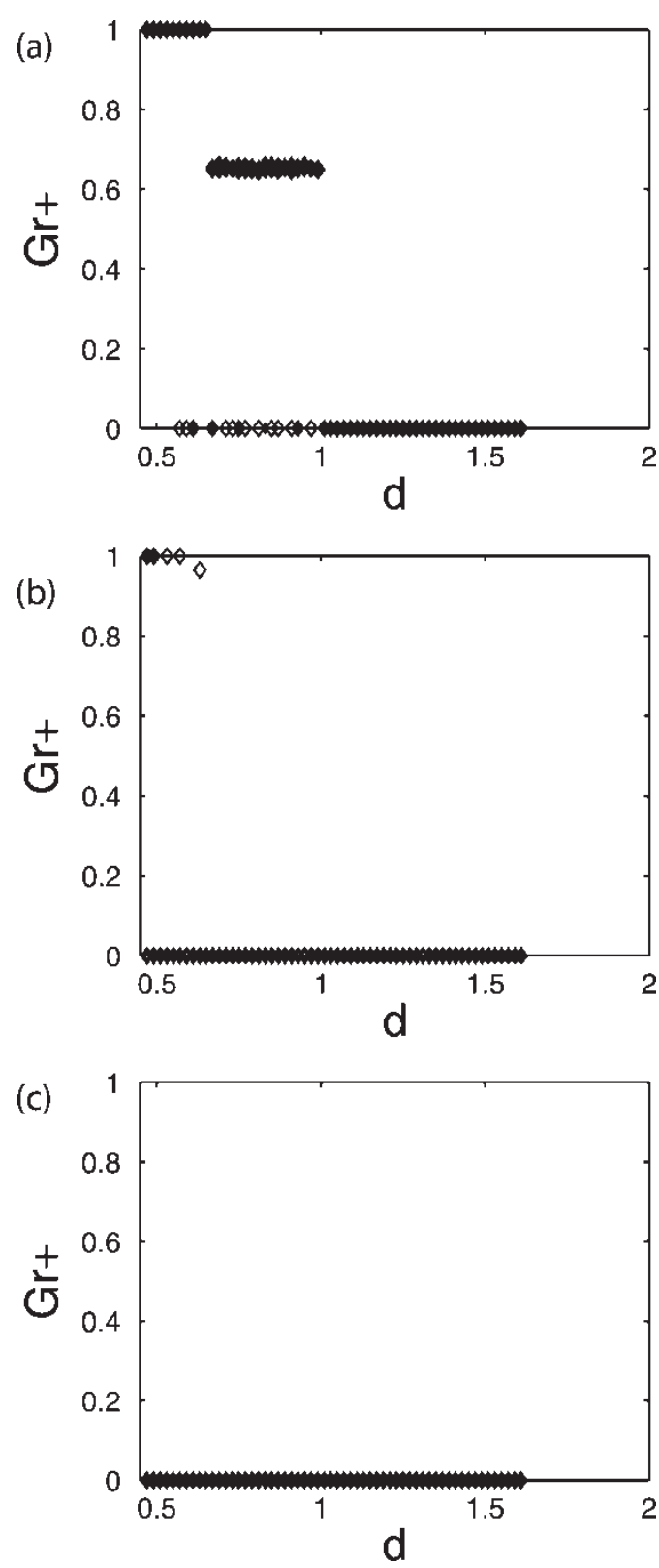

FIGURE 4 (a) Prisoner's Dilemma scenario simulated in a von Neuman neighbourhood according to Table II with parameters $b=1, c=1.5$, and $e=1$. The equilibrium proportion of growth promoters $(\mathrm{Gr}+)$ in the population is depicted as a function of increasing values of $d$. Then simulations have been repeated ten times for each parameter value. Competition for reproduction is fully deterministic and updating is semisynchronous with individual mortality 0.1 . The population was initiated with a proportion of growth promoters of 0.2. (Note that the mean field scenario predicts zero growth promoters for these parameter values.) (b) As in Fig. 4a but based on a Moore neighbourhood. (c) As in Fig. 4a but based on the extended Moore neighbourhood.

scenarios were initialised with frequencies of growth promoters of 0.2 as well as 0.8 since the equilibrium proportion of strategies in this case is sensitive to initial conditions. Furthermore, for some parameter values the Prisoner's Dilemma scenario gave rise to multiple evolutionary outcomes in spite of identical initial proportion of growth promoters. Therefore, ten replications were run for each parameter value in all of the Prisoner's Dilemma scenarios.

\section{The Tomlinson and Bodmer Scenario}

The simulations based on the TB scenario (Table Ia) was investigated for varying values of $b$ while $c$ was kept constant at unity (Fig. 2a-c). The solution of the replicator equation, given in Eq. (2), is $1-c / b$ so as $b$ increases the proportion of growth promoters saturates at 1 (thin solid line in Fig. $2 \mathrm{a}-\mathrm{c}$ ). Figure $2 \mathrm{a}$ shows that the deterministic rule of reproductive competition exhibits drastic discrete shifts in the proportion of growth promoters and nonpromoters (regardless of the level of synchronousity, therefore only semi-synchronous results are shown). Reproductive competition by probabilistic updating (see Fig. $2 b$ and $c$ ), however, show a smooth relationship between $b$ and the dynamic equilibria. Moreover, in contrast to deterministic reproduction the probabilistic reproduction turned out to be sensitive to the level of temporal synchronousity. Semi-synchronous updating gives a confined region of coexistence, as opposed to the asynchronous case, which lies closer to the mean-field result as seen in Fig. $2 \mathrm{~b}$ and $\mathrm{c}$.

Deterministic and probabilistic updating also differ in terms of effect of neighbourhood size. In the deterministic case the Moore and extended Moore neighbourhoods consistently showed lower equilibria of growth promoters for the entire parameter space. This is not the case in simulations applying probabilistic updating. Here, all spatial scenarios gave equlilibria in some cases above and in other cases below the mean-field prediction. In terms of the parameter values for which transitions in the dynamic equilibria occur, these reflect transitions in the local growth dynamics. For example the population level transition for $b=2$ as seen from Fig. 2a, can be explained by a transition in local growth conditions for the exact same parameter value. For a given cell in a von Neumann neighbourhood, which is surrounded by two growth promoters and two non-promoters and with $b>2$, the payoff values of a non-promoter exceeds that of a growth promoter. Now that a non-promoter wins the local competition for proliferation a local triplet of growth promoters can no longer expand (and vice versa for $b<2$ ). Thus, the local transition when $b>2$ give rise to the emergence of a transition on the global level, e.g. for $b$ going from $<2$ to $>2$ the dynamic equilibrium settles on a different dynamic state of a considerably larger proportion of growth promoters (Fig. 2a).

\section{The Hawk-Dove Scenario}

When a growth promoter interacting with another growth promoter is allowed to obtain the additional benefit $(e)$ the scenario becomes identical to a Hawk-Dove game. By letting $e=1,(b-c)=0.5$, and varying $d$, only the payoff of a non-promoter encountering a promoter $(\mathbf{D}-\mathbf{C}$ interaction) is allowed to vary. The thin solid lines on Fig. $3 a-c$ show the mean-field prediction according to Eq. (4). As in the spatial simulations of the TB model, the case of deterministic updating shows the same character- 
istic pattern of drastic transitions in the state of the quasi equilibria. Also, in this case there is no effect of temporal synchronousity on the dynamics when reproduction is deterministic (therefore, only the semi-synchronous realisations are shown in Fig. 3a). Moreover, in line with the findings of Killingback and Doebeli (1996) we find the counter intuitive result that when the Hawk-Dove scenario is implemented with the Moore and extended Moore neighbourhoods there are incidents of increasing levels of co-operation/growth promotion for increasing values of the payoff for non-promoters exploiting promoters (D-C interaction), see Fig. 3a. Interestingly, this is not the case for a von Neumann neighbourhood and such system behaviour is never seen in the probabilistic models Fig. 3b and $\mathrm{c}$, which suggest that the phenomenon may be an artefact of determinism specific to certain neighbourhoods (Moore and extended Moore).

Again, we see that for probabilistic reproduction and asynchronous updating the results tend to approach the mean-field description. However, allowing another element of realism with the semi-synchronous implementation, the discrepancies between the spatial and meanfield description are greatly amplified. This can be seen from the steeper slope and sigmoid shape of the curve in Fig. 3 b compared to Fig. 3c.

\section{The Prisoner's Dilemma Scenario}

The parameter setting describing growth promotion as a truly altruistic act, hence the Prisoner's Dilemma scenario, showed, in contrast to the mean-field result, that clusters of growth promoters, i.e. co-operators, indeed can persist when $c>b$. Figure $4 \mathrm{a}-\mathrm{c}$ show for deterministic updating the final proportion of growth promoters as $\mathrm{d}$ is increased while $e=1$ and $(b-c)=-0.5$ when starting with a population of $20 \%$ growth promoters. As in the above pay off scenarios the implementations with deterministic competition for reproduction show the characteristic discrete transitions in the parameter space. As seen in Fig. 4c a low initial fraction of growth promoters entirely fail to thrive in an extended Moore neighbourhood. In simulations based on the other neighbourhoods the system exhibits multiple evolutionary outcomes with either zero growth promoters or a polymorph population (see replications in Fig. 4a and b). In such case the fate of the growth promoters critically depends on the size of clusters initially present, which in turn determines whether growth promoters gain foothold or go extinct. Figure $5 \mathrm{a}-\mathrm{c}$ show how this effect is less pronounced when the population is initialised with $80 \%$ growth promoters because most likely at least one cluster of the critical size will be present initially. The evolutionary bi-stability has the interesting implication that established (tumour) clusters in this area of the parameter space will be harder to treat subsequent to establishment by changing the costbenefit weighting compared to those cells which are not yet established as clusters. Moreover, this effect could only be observed in the Prisoner's Dilemma scenario with
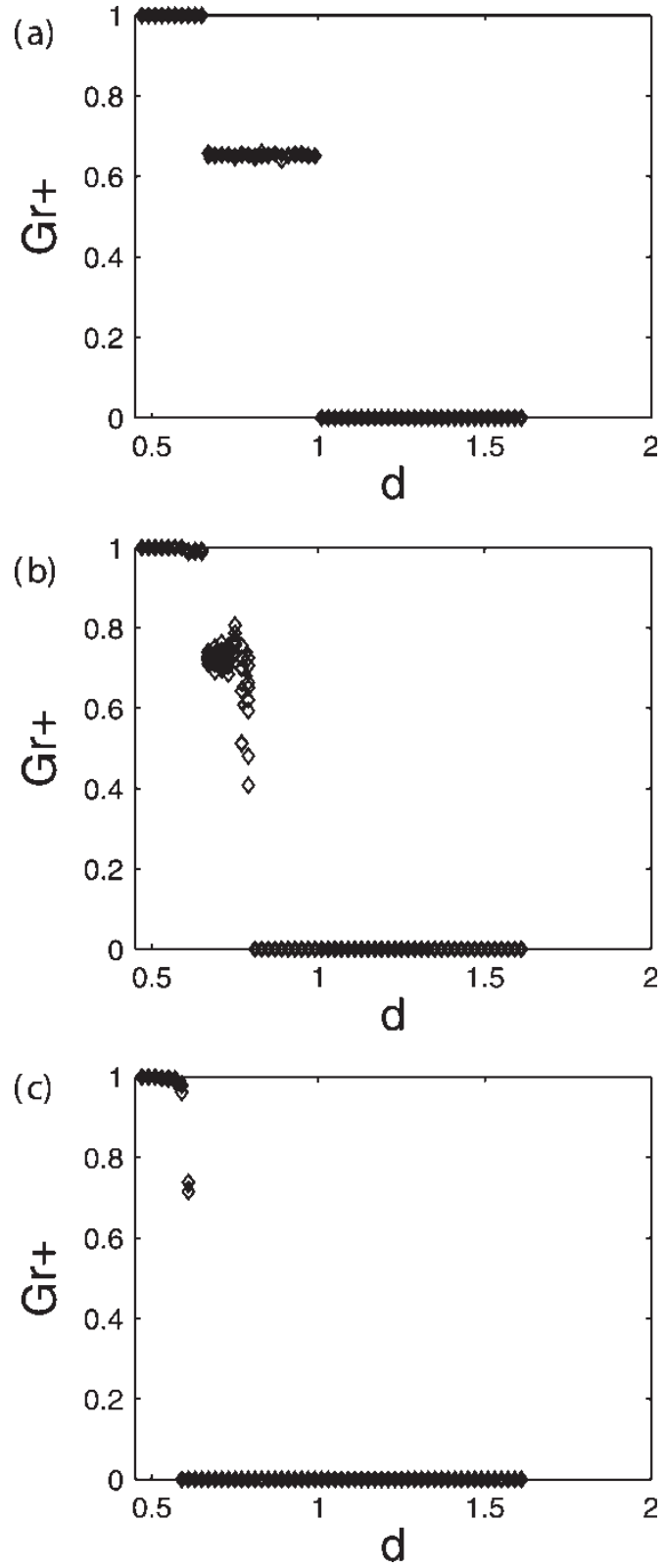

FIGURE 5 (a) As in Fig. 4a but simulations were initiated with a proportion of growth promoters of 0.8. (b) As in Fig. 5a but based on a Moore neighbourhood. (c) As in Fig. 5a but based on the extended Moore neighbourhood.

true altruism $(c>b)$, where spatial correlations are critical for the co-operators. Since the asynchronous and semi-synchronous simulations showed identical dynamics only the latter are shown in Figs. $4 \mathrm{a}-\mathrm{c}$ and $5 \mathrm{a}-\mathrm{c}$.

In order to ascertain the robustness of these results similar simulations were conducted with the following parameter values: $e=1.4,(b-c)=-0.1$ and $e=1.25$, $(b-c)=-0.25$. The same pattern emerged as for the results shown in Figs. 4 and 5 including the drastic transitions among states. The scope for pure co-operation and polymorph states seemed even more pronounced for these parameter values as they represent scenarios closer to the Hawk-Dove scenario. As in Figs. 4 and 5 the system also showed bi-stability in the evolutionary 
outcome for these parameters indicating that sensitivity is a general phenomenon in the parameter region representing the Prisoner's Dilemma scenario.

In contrast to the deterministic cases, when updating was probabilistic, growth promoters always went entirely extinct regardless of the initial conditions and the temporal granularity (results not shown). Hence, both the sequentially asynchronous and semi-synchronous realisations of probabilistic reproduction gave populations exclusively consisting of non-promoters. Local stochasticity as in the probabilistic competition for reproduction allows no possibility for persistence of clusters of "altruistic" growth promoters regardless of the level of synchronicity. In the probabilistic scenario clusters of growth promoters will be invaded by non-promoters. Hence with local stochasticity clusters of growth promoters are no longer immune to exploitation. Therefore, given enough time, such clusters will eventually vanish due to local exploitation in spite of spatial correlations.

\section{DISCUSSION AND CONCLUSION}

As might be expected from previous studies, the spatially extended interaction models showed markedly different results compared to their mean-field counterparts. Space seemed to amplify the proportion of growth promoters in many but not in all of our simulations. However, the spatial simulations were highly sensitive to the specific implementation, which in turn limits the scope for general statements about spatial effects. Because, we consider the probabilistic competition for reproduction and the semisynchronous updating more realistic than the corresponding deterministic and asynchronous representations, the conclusion from previous evolutionary models that space generally favours co-operative strategies does in our view not necessarily apply for populations of cells (Nowak and May, 1992). Indeed, our results indicate that the truly altruistic co-operative strategies thrive poorly among nonaltruists in the domain of a spatial Prisoner's Dilemma scenario except for the (special) case of deterministic competition for reproduction. Even with $80 \%$ growth promoters in the initial population these will eventually be out-competed by non-promoters when realistic local competition is considered.

There are in general, however, significant deviations from the mean-field predictions in our spatial models. These seem to be exacerbated by small neighbourhood sizes: both the deterministic and probabilistic updating scenarios show larger deviations in simulations assuming the von Neumann neighbourhood. Moreover, when probabilistic updating is considered, deviations from the mean-field predictions are highly dependent on whether or not concurrent updates of neighbours are permitted, i.e. semi-synchronous or synchronous updating. Our findings do not fully comply with Hauert's (2002) conclusion, that stochasticity on the level of updating causes equilibrium frequencies to approach the mean-field result.
The biologically motivated probabilistic semi-synchronous scenario showed a pronounced deviation from the mean-field result, in particular in the TB scenario. Indeed, the "window of coexistence" between growth promoting and non-promoting cells is smaller for semi-synchronous than synchronous updating (seen by comparing Figs. $2 b-c$ and $3 b-c)$. Such effects were again more pronounced in the smaller von Neumann neighbourhood. Hence, the degree and level of operation of stochasticity play a non-trivial role concerning the deviation from the mean-field results (see for another example Molofsky et al., 1999).

The effect of neighbourhood size on the deviation from the mean field approximation depends on the level of growth promoters in the population. For example when conditions permit the growth promoting strategy to thrive at a large proportion in the population the smaller neighbourhood size seem to ameliorate further the conditions for growth promoters (see Figs. $2 \mathrm{a}-\mathrm{c}$ and $3 \mathrm{a}-\mathrm{c})$. Thus, longer interaction ranges of the growth factors facilitate the non-promoters' exploitation of the growth promoters, hence it becomes increasingly difficult for the growth promoters to outcompete the nonpromoters completely. Inferentially, a therapeutic intervention inhibiting a putative biochemical growth promoter, (e.g. by lowering its half-life and thereby decreasing the spatial reach) may actually risk to further increase the number of growth promoters. Conversely however, increasing the interaction range of the cells may limit the success of growth promoting strategies.

In both the mean-field and spatial models (with the exception of the deterministic scenario), once across the $b=c$ line then growth promoters will disappear entirely from the population. Thus, increasing sufficiently the cost of promoting growth and being aggressive could cause selection to drive such genotypic strategies out of the population. Furthermore, an interesting implication of the spatial models is that as the benefit of the growth promoter is decreased the proportion of growth promoting cells may change dramatically (Fig. 2b and to a lesser extent Fig. 3b) compared to the mean-field models. These dramatic shifts can occur even when $b>c$. Such selective collapse of a growth promoting genotype cannot be excluded as a possible scenario explaining the phenomenon of spontaneous regression. In some cases the organism's immune system could be thought to naturally increase the efficiency towards cancerous cells, hence increasing c. However, it should be noted that a number of other explanations for this phenomenon have been suggested. The most common cases of spontaneous regression occur for certain malignant tumours in very young children $(<1$ year of age) and presumably correlate with a general decrease in the production of growth factors (Papac, 1996). Similarly, breast carcinomas that are dependent on hormonal stimulation can regress at the onset of menopause (Papac, 1996).

Recent advances in the understanding of genetic abnormalities in cancer cells have led to an exciting development of new treatment modalities that might 
directly affect the values of $c$ (ost) and $b$ (enefit). When surgery is inadequate or impossible, cancer cells have traditionally been hit with agents that basically target any living cell that has a very high metabolic rate and is rapidly dividing. Today, drugs or agents are being exquisitely designed for molecular specificity (or genotype specificity) by targeting various signalling molecules and pathways that are deregulated in cancer cells. These agents still have to overcome two major problems of cellular specificity (since these signalling pathways are also present in normal cells) and the power of selection and evolution leading to relapse and development of a resistant clone (Greaves, 2000; Gorre et al., 2001). Nevertheless, these new treatment modalities offer some intriguing possibilities of modifying $c$ and $b$. Although, it still needs a lot of development, an example in which the cost of having a given genotype could be dramatically increased is the use of replication-competent viruses (Alemany et al., 2000). Furthermore, recent work with the re-population of tumour reactive $T$-cells that can induce metastatic regression, would here correspond to an increase in the cost of being a specific cell type (Dudley et al., 2002). An example where the value of $b$ might be modified could be the use of angiogenesis inhibitors (Carmeliet and Jain, 2000). When a patient present with a tumour, the new blood vessels are already established. Thus, from an evolutionary dynamics point of view, angiogenesis inhibitors are probably mainly of use when applied in a preventive setting rather than when used as therapeutics.

This study represents an initial step in making spatial tumour models which account for evolutionary effects such as local frequency-dependent selection. In previous spatial models the autocrine and paracrine growth factors have been seen as determining growth parameters. The cells inside a tumour should be regarded as entities that are subject to strong selection and interaction amongst each other and should not be modelled as static units inside a tumour. Since the first models of tumour growth such as the early work of Thomlinson and Gray (1955) and the model of layered growth by Greenspan (1972) to the present state of the art models including the cellular automaton based models (Qi et al., 1993; Kansal et al., 2000; Moreira and Deutsch, 2002), and models based on differential equations and diffusion (Michelson and Leith, 1991; Chaplain et al., 2001; Breward et al., 2002; Jackson, 2002; Sherratt and Chaplain, 2002), there seem to have been surprisingly little attention to the kind of evolutionary dynamics generated by frequencydependent interactions among cells. Such effects should also be considered if tumour models are to become more realistic, since an inherent property of cancerous growth is extremely high turnover rates, in turn facilitating shortterm adaptation and local frequency-dependent selection among cells. With most of the pharmaceutical industry focussing on "designer" drugs targeting various signalling pathways (or genotypes) altered in various tumour cells, a better understanding of tumour evolutionary dynamics and spatial heterogeneity seems more important than ever (Gonzales-Garcia et al., 2002). An underestimation of the power of selection and evolution can be detrimental in connection to treatment and prognosis (Gorre et al., 2001). The coupling of population dynamics with evolutionary dynamics, which has been a major focus of research in ecology, is equally important in the description of phenomena such as internal tumour dynamics.

\section{Acknowledgements}

We are grateful to T. Helvik, M. Gade, and J. Koella for valuable and motivating discussions and to two anonymous reviewers for helpful comments on an earlier version of the manuscript. This work was possible through the kind hospitality to LAB and DJTS of the Newton Institute in November 2001 at the 'Individual to Collective Behaviour in Biological Systems' programme.

\section{References}

Alemany, R., Balague, C. and Curiel, D.T. (2000) "Replicative adenoviruses for cancer therapy", Nature Biotechnology 18(7), $723-727$.

Axelrod, R. and Hamilton, W.D. (1981) "The evolution of co-operation", Science 211, 1390-1396.

Bach, L.A., Bentzen, S., Alsner, J. and Christiansen, F.B. (2001) "An evolutionary-game model of tumour-cell interactions: possible relevance to gene therapy", European Journal of Cancer 37, 2116-2120.

Binmore, K.G. (1996) Fun and Games, 1st ed. (Houghton Mifflin, Boston, MA).

Boehm, T., Folkman, J., Browder, T. and O'Reilly, M. (1997) "Antiangiogenic therapy of experimental cancer does not induce acquired drug resistance", Nature 390, 404-407.

Boyer, M.J. and Tannock, I.F. (1998) The Basic Science of Oncology, 3rd ed. (McGraw-Hill, New York).

Breward, C.J.W., Byrne, H.M. and Lewis, C.E. (2002) "The role of cellcell interactions in a two-phase model for avascular tumour growth", Journal of Mathematical Biology 45, 125-152.

Carmeliet, P. and Jain, R.K. (2000) "Angiogenesis in cancer and other diseases", Nature 407, 249-257.

Challis, G.B. and Stam, H.J. (1990) "The spontaneous regression of cancer", Acta Oncologica 29(5), 545-550.

Chaplain, M.A., Ganesh, M. and Graham, I.G. (2001) "Spatio-temporal pattern formation on spherical surfaces: numerical simulation and application to solid tumour growth", Journal of Mathematical Biology 42(5), 387-423.

Dieckmann, U. (1997) "Can adaptive dynamics invade?", Trends in Ecology and Evolution 12(4), 128-131.

Dudley, M.E., Wunderlich, J.R., Robbins, P.F., Yang, J.C., Hwu, P., Schwartzentruber, D.J., Topalian, S.L., Sherry, R., Restifo, N.P., Hubicki, A.M., Robinson, M.R., Raffeld, M., Duray, P., Seipp, C.A., Rogers-Freezer, L., Morton, K.E., Mavroukakis, S.A., White, D.E. and Rosenberg, S.A. (2002) "Cancer regression and autoimmunity in patients after clonal repopulation with antitumor lymphocytes", Science 298(5594), 850-854.

Durrett, R. and Levin, S. (1994) "The importance of being discrete (and spatial)", Theoretical Population Biology 46, 363-394.

Gonzales-Garcia, I., Sole, R.V. and Costa, J. (2002) "Metapopulation dynamics and spatial heterogeneity in cancer", Proceeding of the National Academy of Sciences 99(20), $13085-13089$.

Gorre, M.E., Mohammed, M., Ellwood, K., Hsu, N., Paquette, R., Rao, P.N. and Sawyers, C.L. (2001) "Clinical resistance to sti-571 cancer therapy caused by bcr-abl gene mutation or amplification", Science 293(5531), 876-880. 
Greaves, M. (2000) Cancer: The Evolutionary Legacy (Oxford University Press, Oxford).

Greenspan, H.P. (1972) "Models for the growth of a solid tumour by diffusion", Studies of Applied Mathematics 51, 317-340.

Hanahan, D. (2000) "The hallmarks of cancer", Cell 100(1), 57-70.

Hauert, C. (2001) "Fundamental clusters in $2 \times 2$ spatial games", Proceedings of the Royal Society of London Series B 268, 761-769.

Hauert, C. (2002) "Effects of space in $2 \times 2$ games", International Journal of Chaos and Bifurcation 12(7), 1531-1548.

Herz, A.V.M. (1994) "Collective phenomena in spatially extended systems", Journal of Theoretical Biology 169, 65-87.

Hofbauer, J. and Sigmund, K. (1988) The Theory of Evolution and Dynamical Systems (Cambridge University Press, Cambridge).

Huberman, B.A. and Glance, N.S. (1993) "Evolutionary games and computer simulations", Nature 90, 7716-7718.

Jackson, T.L. (2002) "Vascular tumor growth and treatment: Consequences of polyclonality, competion and dynamic vascular support", Journal of Mathematical Biology 44, 201-226.

Kansal, A.R., Torquato, S., IV, G.R.H., Chiocca, E.A. and Deisboeck, T.S. (2000) "Simulated brain tumour growth dynamics using a threedimensional cellular automaton", Journal of Theoretical Biology 203, 367-382.

Killingback, T. and Doebeli, M. (1996) "Spatial evolutionary game theory: Hawks and doves revisited", Proceedings of the Royal Society of London Series B 263, 1135-1144.

Koella, J. (2000) "The spatial spread of altruism versus the evolutionary response of egoists", Proceedings of the Royal Society of London Series B 267, 1979-1985.

Lindgren, K. and Nordahl, M.G. (1994) "Evolutionary dynamics of spatial games", Physica D 75, 292-309.

Maynard-Smith, J. (1982) Evolution and the Theory of Games, 1st ed. (Cambridge University Press, Cambridge).

Maynard-Smith, J. and Price, G. (1973) "The logic of animal con ict", Nature 246, 7716-7718.

McGlade, J. (1999) Advanced Ecological Theory (Blackwell Science, London).

Michelson, S. and Leith, J. (1991) "Autocrine and paracrine growth factors in tumor growth: a mathematical model", Bulletin of Mathematical Biology 53(4), 639-656.

Molofsky, J., Durrett, R., Dushoff, J., Griffeath, D. and Levin, S. (1999) "Local frequency dependence and global coexistence", Theoretical Population Biology 55(3), 270-282.

Mora, J., Cheung, N.K.V. and Gerald, W.L. (2001) "Genetic heterogeneity and clonal evolution in neuroblastoma", British Journal of Cancer 85(2), 182-189.

Moreira, J. and Deutsch, A. (2002) "Cellular automaton models of tumor development: a critical review", Advances in Complex Systems $\mathbf{5}(2-3), 247-267$.

Nakamuru, M., Matsuda, H. and Iwasa, Y. (1997) "The evolution of co-operation in a lattice-structured population", Journal of Theoretical Biology 184, 65-81.

Nowak, M.A. and May, R. (1992) "Evolutionary games and spatial chaos", Nature 18(359), 826-829.

Nowell, P.C. (1976) "The clonal evolution of tumour cell populations", Science 194, 23-28.

Onyango, P. (2002) "Genomics and cancer", Current Opinion in Oncology 14, 79-85.

Page, K., Nowak, M. and Sigmund, K. (2000) "The spatial ultimatum game", Proceedings of the Royal Society of London Series B 267, $2177-2187$.

Papac, R.K. (1996) "Spontaneous regression of cancer", Cancer Treatment Review 22(6), 395-423.

Qi, A., Zhen, X., Du, C. and An, B. (1993) "A cellular automation model of cancerous growth", Journal of Theoretical Biology 161(1), 1-12.

Rapoport, A., Guyer, M.J. and Gordon, D.G. (1976) The $2 \times 2$ Game, 1st ed. (University of Michigan Press, Michigan).

Riechert, S.E. and Hammerstein, P. (1983) "Game theory in the ecological context", Annual Review of Ecology and Systematics 14, $377-409$.

Sherratt, J.A. and Chaplain, M.A.J. (2002) "A new mathematical model for avascular tumour growth", Journal of Mathematical Biology 43, $291-312$.

Svenstrup, M. and Christiansen, F.B. (2000) "Biker's dilemma: Co-operation between foes", Selection 1-3, 147-152.

Szabo, G. and Hauert, C. (2002) "Phase transitions and volunteering in spatial public goods games", Physical Review Letters 89(11), 118101.
Thomlinson, R.H. and Gray, L.H. (1955) "Histological structures of some human lung cancers and the possible implications for radiotherapy", British Journal of Cancer 9, 539-549.

Tomlinson, I.P. (1997) "Game-theory models of interactions between tumour cells", European Journal of Cancer 33(9), 1495-1500.

Tomlinson, I.P. and Bodmer, W.F. (1997) "Modelling consequences of interactions between tumour cells", British Journal of Cancer 75, $157-160$.

Tomlinson, I.P.M. (1993) "Mutations, evolutionary theory and cancer", Trends in Ecology and Evolution 8(3), 107-110.

Tomlinson, I.P.M., Novelli, M.R. and Bodmer, W.F. (1996) "The mutation rate and cancer", Proceedings of the National Academy of Science of the United states of America 93(25), 14800-14803.

Wolfram, S. (1984) "Cellular automata as models of complexity", Nature 311(4), 419-424.

\section{APPENDIX}

Generation Turn Over:

procedure Outer iterative loop

begin

Initialise population on lattice

for $g=0$ to $g=$ maxgeneration do

Payoff update

Mortality

Competitive Reproduction

end

end

\section{A.1 Payoff Update:}

procedure Payoff updating

begin

for each individual $i$ in lattice do

fitness $i=0$

for each neighbour $j$ of $i$ do

Get payoff from pairwise interaction $[i, j]$ according to payoff table

fitness $(i)=$ fitness $(i)+$ payoff

end

end

end

\section{A.2 Mortality:}

procedure Asynchronous mortality

begin

remove individual $i$ at random location

return

end procedure Semi-synchronous mortality

begin

for each individual $i$ in lattice do

if mortality probability $>$ PseudoRandom $[0 ; 1]$

then

remove $i$

return 


end
end
end

\section{A.3 Reproduction:}

procedure Reproduction by competition begin

for each position $p$ in lattice do if $p$ is void then

if competition $=$ deterministic then select neighbour $j$ with highest fitness (if tie select randomly)

\author{
copy instance of $j$ into location $p$ \\ end
}

if competition $=$ probabilistic then total fitness $=0$

for each neighbour $n$ do

total fitness $=$ total fitness + fitness $(n)$ end

select neighbour $j$ with probability $=$ fitness (j)/ total fitness end

copy instance of $j$ into location $p$

end

end

end 


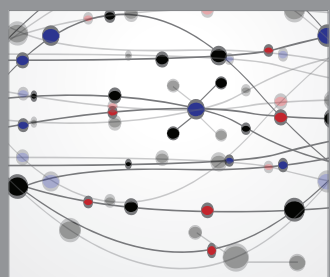

The Scientific World Journal
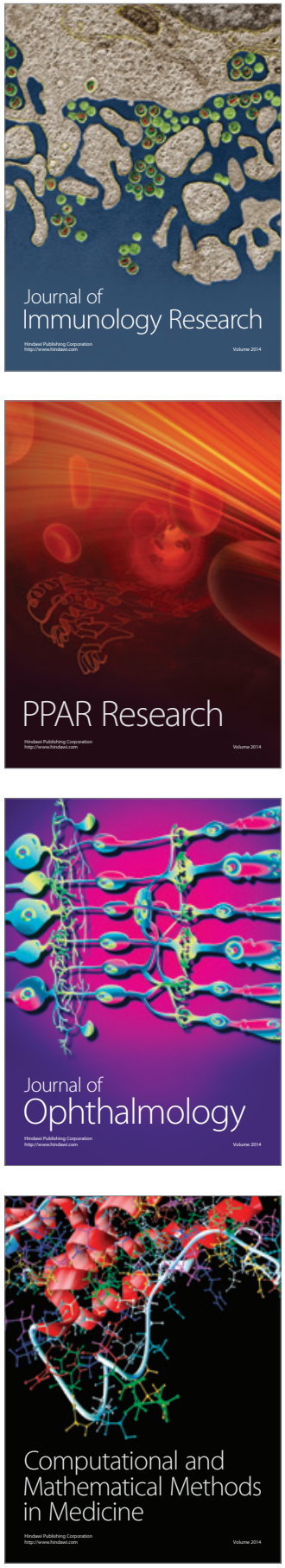

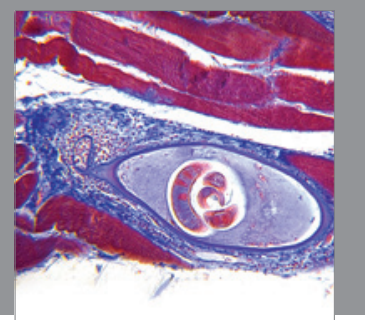

Gastroenterology

Research and Practice
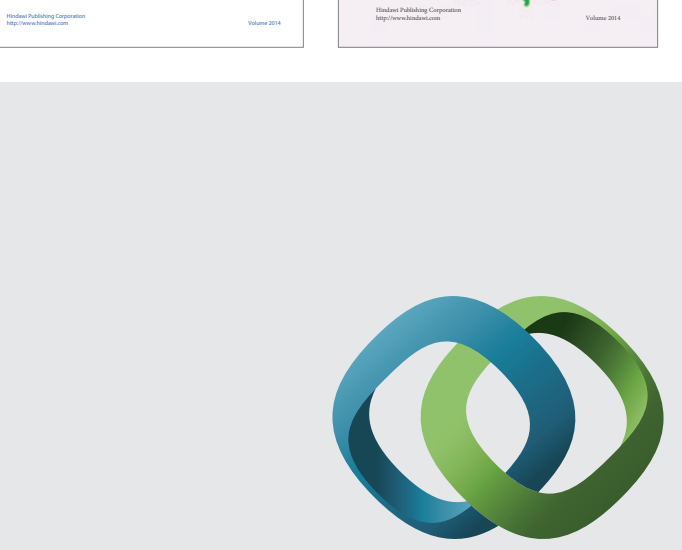

\section{Hindawi}

Submit your manuscripts at

http://www.hindawi.com
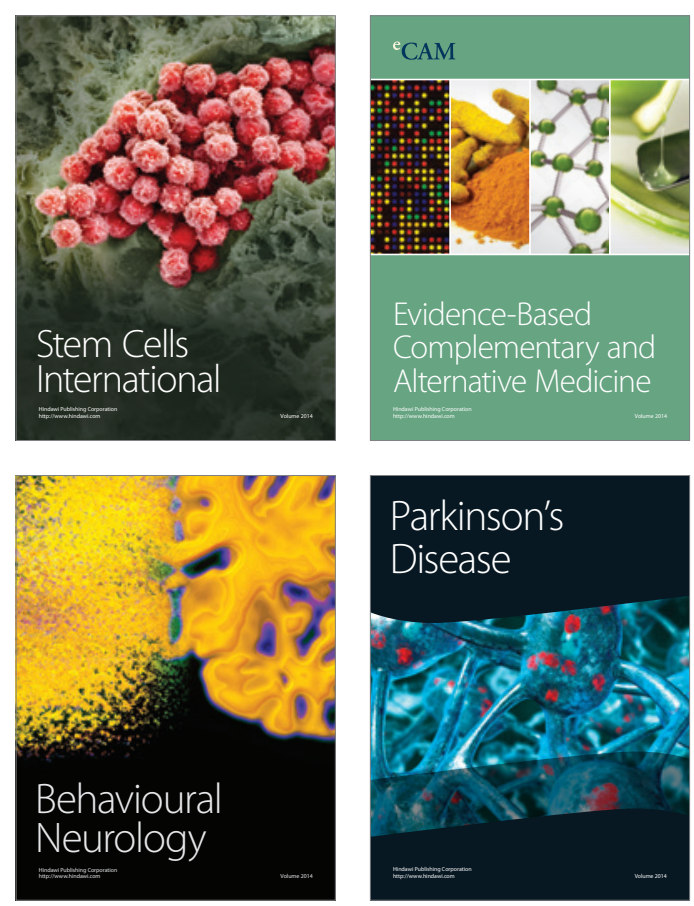

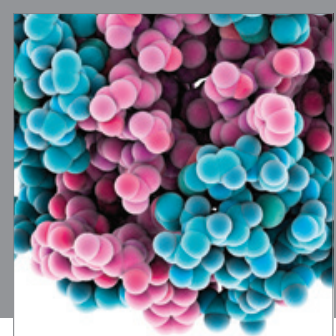

Journal of
Diabetes Research

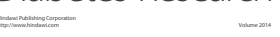

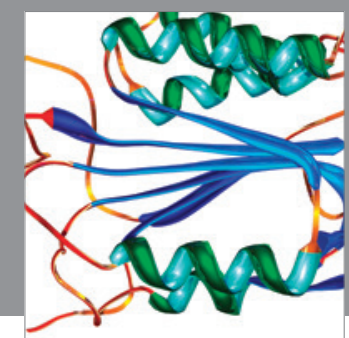

Disease Markers
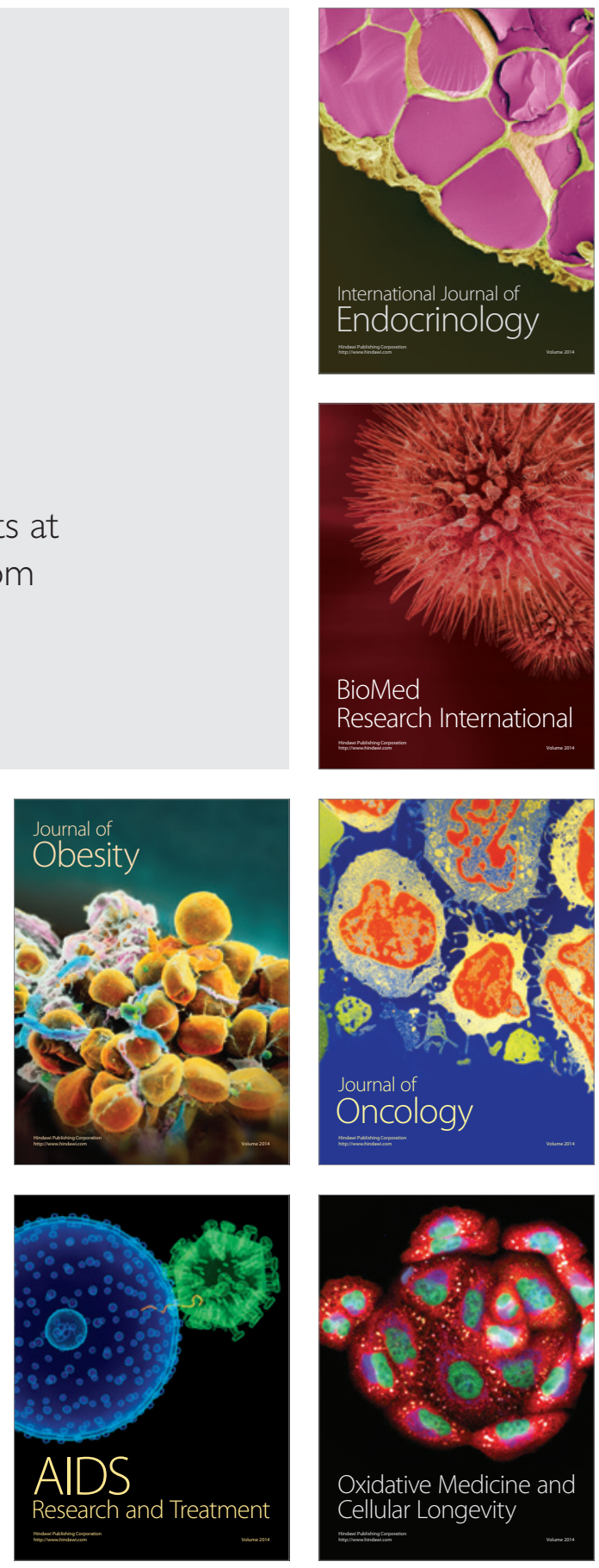